\title{
Early Standard Model physics and early discovery strategy in ATLAS
}

\author{
J. Grosse-Knetter ${ }^{\mathrm{a}}$ on behalf of the ATLAS collaboration,

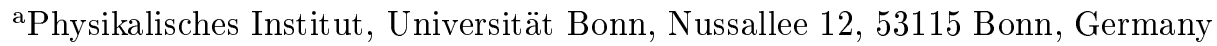

In 2008 the LHC will open a new energy domain for physics within the Standard Model and beyond. The physics channels which will be addressed by the ATLAS experiment in the initial period of operation will be discussed. These include Standard Model processes such as $W / Z$ production and early top measurements. This will be followed by a description of the searches for a low-mass Higgs boson, new heavy di-lepton resonances, and Supersymmetry, for which a striking signal might be observed after only a few months of data taking.

\section{INTRODUCTION}

ATLAS is one of the two multi-purpose detectors for new physics in the $\mathrm{TeV}$ mass range which are being constructed for operation at the LHC [1,2]. The LHC will collide two proton beams at a centre-of-mass energy of $14 \mathrm{TeV}$. LHC is scheduled to provide collisions at $14 \mathrm{TeV}$ from summer 2008 on. The initial delivered peak luminosity is expected to be below $10^{33} \mathrm{~cm}^{-2} \mathrm{~s}^{-1}$, i.e. up to $1 \mathrm{fb}^{-1}$ integrated luminosity per experiment until the end of 2008. In the course of 2009, LHC is anticipated to deliver $1 \ldots 2 \cdot 10^{33} \mathrm{~cm}^{-2} \mathrm{~s}^{-1}$, i.e. up to $10 \mathrm{fb}^{-1}$ until the end of 2009. This report will thus focus on physics which is accessible with a few $\mathrm{fb}^{-1}$ or less.

ATLAS comprises several layers, extending from the Inner Detector (ID), to the superconducting solenoid magnets with a $2 \mathrm{~T}$ field, calorimeters, muon chambers, and an air toroid with a field of up to $4 \mathrm{~T}$. The ID combines tracking straw tubes in the outer transition-radiation tracker with silicon microstrip detectors in the middle and silicon pixel detectors in the innermost component. The calorimeter is composed of liquid argon(LAr)-lead, LAr-tungsten or LArcopper cells for both electromagnetic and parts of the hadronic calorimeter, and the barrel hadronic steel-scintillator tile calorimeter. The muon spectrometer combines drift tubes and cathode strips for precision tracking with resistive plate chambers and thin gap chambers for triggering. A detailed description of the current status of ATLAS can be found elsewhere [3].

\section{STANDARD MODEL MEASURE- MENTS}

Standard Model (SM) processes at LHC have a cross-section which is one to two orders of magnitudes larger than at Tevatron, so that ATLAS expects thousands if not millions of events with the production of $W, Z$ and $t$ (taking selection efficiency and detector acceptance into account) already during low-luminosity operation. Consequently, statistical errors in most measurements will be small or even negligible, and systematic effects must be analysed thoroughly.

\subsection{Underlying event}

Soft hadron interactions will be amongst the first processes being studied at ATLAS due to their large cross-section. This includes measurements of the level to which underlying events occur, providing important information for the analysis of other physics processes. Although the properties of underlying events have extensively been studied at Tevatron [4], the extrapolation of such measurement to the LHC centre-of-mass energy has large uncertainties between available models [5], as shown in figure 1. Thus a measurement of properties of charged particles in the region transverse to the leading jet, the region dominated by particles coming from the underlying event, as function of the transverse momentum $p_{T}$ of the leading jet will be used to tune Monte Carlo generators accordingly. 


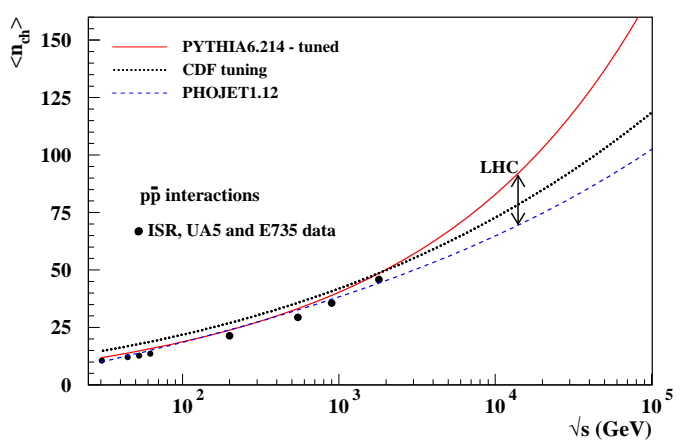

Figure 1. Average charged particle multiplicity in non-single-diffractive $p \bar{p}$ events [5].

\section{2. $W$ and $Z$ production}

During the early LHC phase at low luminosity ATLAS will trigger on large samples of SM candles such as production of $Z$ and $W$ bosons with $Z \rightarrow \ell \ell$ and $W \rightarrow \ell \nu$, where $\ell=e, \mu$, but also $\tau$ is of interest, in particular when it decays hadronically.

Events with $Z \rightarrow \ell \ell(\ell=e, \mu)$ will be essential for the final energy and momentum scale calibration, while the calibration of missing transverse energy $E_{T}^{\text {miss }}$ will use $W \rightarrow \mu \nu$ and $Z \rightarrow \tau \tau$. In addition, the understanding of $W+$ jets and $Z+$ jets processes is important for precise knowledge of the background for searches. Finally, measuring the $W \rightarrow \tau \nu$ cross section by selecting events triggering on hadronic $\tau$-decays and cuts on $E_{T}^{\text {miss }}$ will allow a validation of $\tau$ identification algorithms needed for searches.

In addition, ATLAS will collect rapidly sufficient statistics to improve measurements of the $W$ mass which is described in detail elsewhere [6].

\section{3. $W$ rapidity}

The production of $W$ bosons at LHC is, at small rapidity, dominated by partons with small momentum fraction $x$ entering the process $q \bar{q} \rightarrow$ $W$ (at leading order). Therefore, mostly sea quarks contribute, so that the uncertainties in the production cross section and the $W$ rapidity dis-

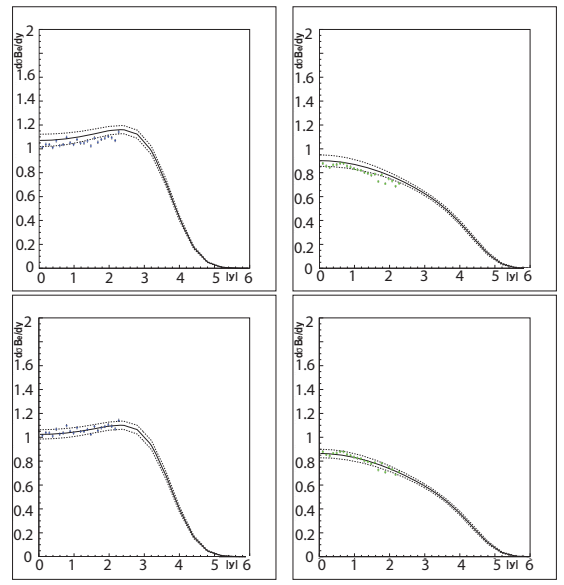

Figure 2. Top row: $e^{+}$and $e^{-}$rapidity spectra generated from CTEQ6.1 PDFs, which have been passed through the ATLAS fast detector simulation and corrected back to generator level using ZEUS PDFs, compared to the analytic prediction using ZEUS PDFs. Bottom row: the same lepton rapidity spectra compared to the analytic prediction after including these lepton pseudo-data in the ZEUS PDF fit [8].

tribution of approx. $7 \%$ is dominated by that on the gluon PDF $[7,8]$. ATLAS can thus constrain further the gluon PDF by measuring the rapidity spectrum of the $W$ decay products.

An ATLAS study based on pseudo-data corresponding to approx. $100 \mathrm{pb}^{-1}$ including $4 \%$ systematic uncertainty [8] shows that a few simple cuts are sufficient to suppress the background in the pseudo-rapidity range $|\eta|<2.4$, where tracking is fully efficient. This analysis subsequently uses the rapidity-spectra of $e^{+}$and $e^{-}$ from $W$ decays (see figure 2) as additional input to ZEUS PDF fits, resulting in a reduced error in the parameter $\lambda$ of the gluon PDF at input scale, $x g(x) \propto x^{\lambda}: \quad \lambda=-0.199 \pm 0.046$ before, and $\lambda=-0.181 \pm 0.030$ after including the ATLAS pseudo-data, corresponding to a reduction in the error by $35 \%$. 


\subsection{Di-boson production}

The production of di-bosons at LHC allows to probe triple-gauge couplings, testing SM predictions for charged couplings like $W W \gamma$ and $W W Z$ or to search for SM-forbidden pure neutral couplings like $Z Z Z$ or $Z Z \gamma$. For example, the production of a $W Z$ pair is sensitive only to $W W Z$ coupling. It has a clean signal in all decay channels eee $\nu, e e \mu \nu, e \mu \mu \nu$, and $\mu \mu \mu \nu$, such that requiring three charged leptons above a certain $p_{T}$ and a minimum $E_{T}^{\text {miss }}$ largely suppresses any background. At a luminosity of $1 \mathrm{fb}^{-1}$, approx. 75.7 signal events with 7.5 background events coming mostly from $Z+$ jets processes are expected, i.e. $W Z$ production will be detected with a significance of $S / \sqrt{B}=27.7$.

\subsection{Early $t \bar{t}$ measurements}

Top quark pair production at LHC will be dominated by the gluon-gluon fusion process, which together with the higher centre-of-mass energy results in a cross-section which is approx. a factor 100 higher than at Tevatron, whereas the background is expected to rise only by a factor of 10 . Consequently, a number of top-quark measurements can be performed by ATLAS with no lack of statistics and little background. For example, the semi-leptonic decay channel $t \bar{t} \rightarrow \ell \nu+4$ jets is selected requiring one lepton, $E_{T}^{\text {miss }}$ and four high- $p_{T}$-jets, of which two have to be tagged as b-jets, resulting in almost negligible background. Such events will be used for top mass, crosssection, and decay mechanism measurements, all of which will eventually be limited by systematic uncertainties $[9,10]$.

Given that in the initial phase of ATLAS operation b-tagging might not be well understood, the b-tag requirement can be dropped from the selection of the semi-leptonic channel. The hadronically decaying top is then identified by the three jets with the highest sum in $p_{T}$. This selection still leaves a reasonable signal peak above a modest background, see figure 3 . Such a data set will also be used to test and understand the b-tagging further and to extract clean samples of $W$ bosons from the decay $t \rightarrow b W$, which are then used to study the jet energy scale in the hadronic decay of the resp. $W$ boson [11].

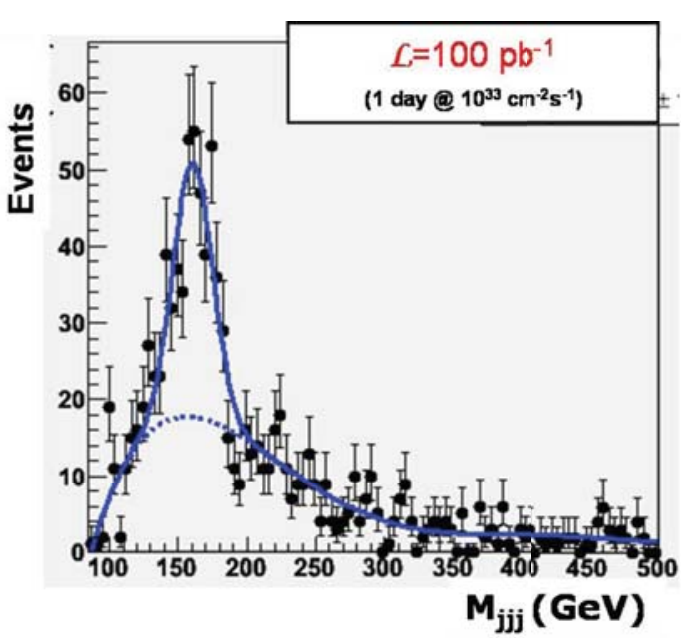

Figure 3. Invariant mass of the three jets with highest $\Sigma p_{T}$ assumed to belong to a hadronically decaying $W$ from $t \rightarrow b W$ in $t \bar{t}$ production. The dashed line indicates the contribution from combinatorial and other background sources.

\section{SEARCHES}

\subsection{Supersymmetry}

SUSY searches are amongst the most promising for early data taking with ATLAS: assuming a squark or gluino mass of around $1 \mathrm{TeV}$, SUSY events will be seen at a rate of about 100 events per day at $\mathcal{L}=10^{33} \mathrm{~cm}^{-2} \mathrm{~s}^{-1}$ due to production via strongly coupling squarks and gluinos. If these are heavier than sleptons, gauginos etc., cascade decays down to the lightest SUSY particle (LSP) will result in long decay chains. The large mass differences between SUSY states produces many high- $p_{T}$ objects (leptons, jets) that can be observed easily. If R-parity is conserved, the LSP is stable and SUSY can be identified by a large $E_{T}^{\mathrm{miss}}$ signature. A more refined variable combines the $p_{T}$ of the leptons and jets from the decay cascade and the $E_{T}^{\text {miss }}$ caused by the escaping LSP into $M_{\text {eff }}=\sum_{\text {leptons,jets }}\left|p_{T}\right|+E_{T}^{\text {miss }}$. Apart from the more robust selection, an additional benefit is that $M_{\text {eff }}$ relates to the SUSY mass scale [12]. 


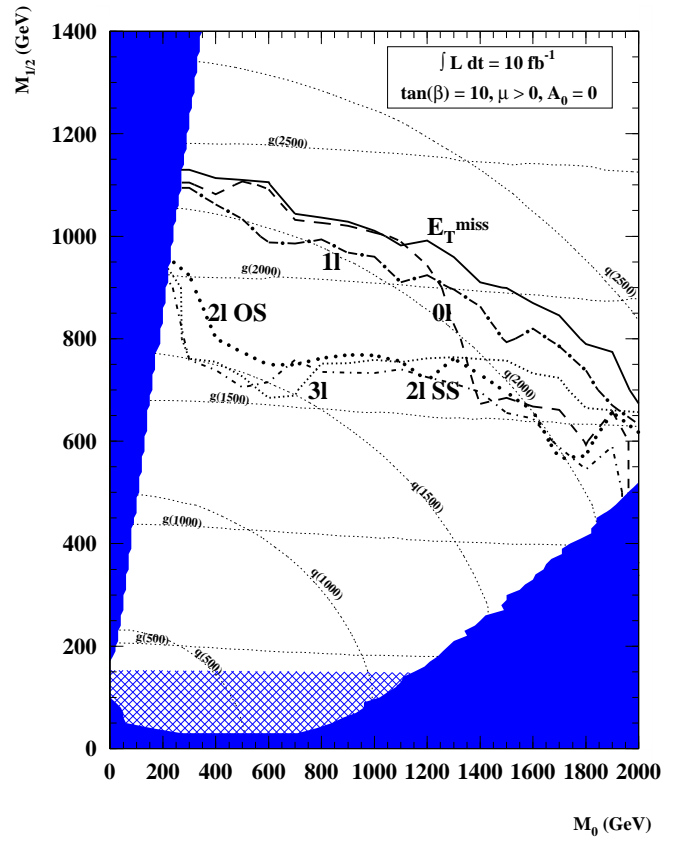

Figure 4. $5 \sigma$ discovery potential in the $M_{0}-M_{1 / 2}$ plane for mSUGRA models with $\tan \beta=10$, $\mu>0$ and $A_{0}=0$ for $10 \mathrm{fb}^{-1}$. Bold curves correspond to: $E_{T}^{\text {miss }}$ channel (full curve), nolepton channel (dashed curve), one, two opposite sign (OS), same sign (SS) and three lepton channel (dotted curves). Light curves correspond to squark and gluino iso-mass contours (masses in $\mathrm{GeV})$ [12].

Modelling the background in Monte Carlo needs a good understanding of high- $p_{T}$ jets, a region in which newer Monte Carlo generators using matrix-element/parton-shower matching are expected to give a more reliable prediction than previous generators only based on parton showers. The largest physics background is neutrino emission (e.g. $Z \rightarrow \nu \nu$ ), thus a good understanding of $E_{T}^{\text {miss }}$ is necessary. It was demonstrated that e.g. $Z \rightarrow \mu \mu$ events can be used to model the $E_{T}^{\text {miss }}$-spectrum from $Z \rightarrow \nu \nu$ events.

Using the most efficient and general signa- ture, that of $E_{T}^{\text {miss }}$, a discovery of SUSY is already possible with an integrated luminosity of $1 \mathrm{fb}^{-1}[12,13]$. Using $M_{\mathrm{eff}}$ as signature alternatively, the the discovery power is similar, depending on the number of leptons that is required, see figure 4 .

\subsection{Di-lepton resonances}

Di-lepton resonances in the $1 \mathrm{TeV}$ mass range are expected from a number of models, such as new heavy gauge bosons predicted by GUT or dynamical electroweak symmetry breaking, Kaluza-Klein excitations or Randall-Sundrum gravitons [14]. The ATLAS discovery potential was investigated for a heavy $Z^{\prime}$ in the CDDT parametrisation [15], showing a large mass peak above low background, mostly comprising (irreducible) Drell-Yan events, or $Z Z, Z W, W W$, and $t \bar{t}$ production. This initial study focused on $Z^{\prime} \rightarrow e^{+} e^{-}$since performance is expected to be better than with $Z^{\prime} \rightarrow \mu^{+} \mu^{-}$due to better resolution of the calorimeter at high energy. The resulting discovery range for $400 \mathrm{pb}^{-1}$ is shown in figure 5 demonstrating a good reach into the $\mathrm{TeV}$ mass region already at an early stage.

\subsection{Standard Model Higgs}

The most important channels for SM Higgs searches in the LEP-favoured low-mass region are Vector Boson Fusion (VBF) production with decays $H \rightarrow \tau \tau$ and $H \rightarrow W W$, gluon fusion production with $H \rightarrow \gamma \gamma$ and, at higher mass, $H \rightarrow 4 \ell$ [16]. Again, the understanding of detector performance and SM background processes as discussed above at an early stage is important; e.g. for VBF processes the production of $W$ or $Z$ with extra jets is of particular interest.

The SM Higgs can be discovered with at least a $5 \sigma$ signal in the mass range $m_{H}>120 \mathrm{GeV}$ with an integrated luminosity of $10 \mathrm{fb}^{-1}[17]$ as shown in figure 6 . It should be noted that a combination of several analyses using different Higgs decay and production modes may be necessary since one mode alone does not necessarily reach beyond the $5 \sigma$ level. The highest significances are reached at a Higgs mass around $160 \mathrm{GeV}$, where a discovery is possible with less than $10 \mathrm{fb}^{-1}$. 


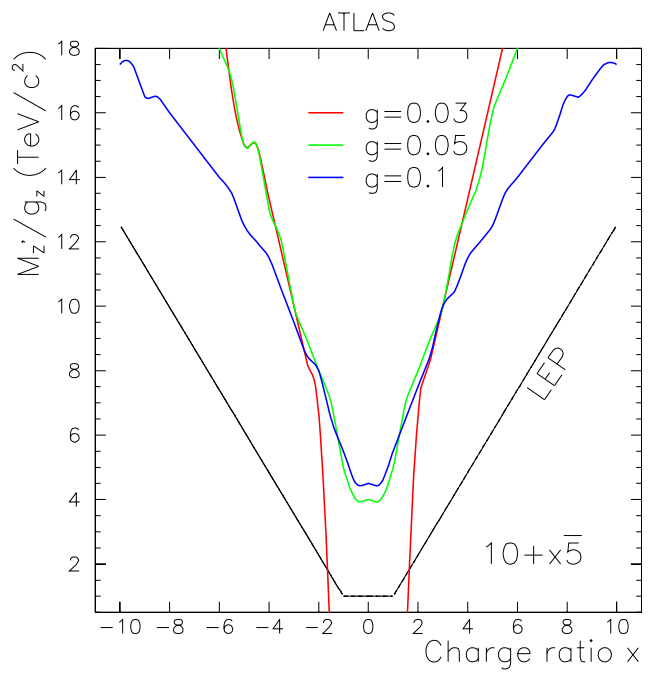

Figure 5. Discovery potential of ATLAS for a $Z^{\prime}$ in one of four classes of the CDDT parametrisation with $400 \mathrm{pb}^{-1}$ of data in the $M_{Z^{\prime}} / g_{Z^{-}} x$ plane, where $M_{Z^{\prime}}$ is the $Z^{\prime}$ mass, $g_{Z}$ its global coupling strength and $x$ the relative coupling strength to fermions [15].

\section{Summary}

First physics data taking with ATLAS at $14 \mathrm{TeV}$ will start summer 2008, which will allow to study a large variety of processes on a few $\mathrm{fb}^{-1}$. This includes Standard Model interactions like underlying event, minimum bias and QCD processes, $\mathrm{W}, \mathrm{Z}$ production, di-boson production and top pair production, as well as first searches such as probing SUSY in the $1 \mathrm{TeV}$ mass scale, searching for di-lepton resonances and, possibly, discovery of the SM Higgs boson.

\section{REFERENCES}

1. ATLAS Technical Proposal for a GeneralPurpose pp Experiment at the LHC at CERN, CERN/LHCC/94-43 (1994).

2. ATLAS Detector and Physics Performance, CERN/LHCC/99-15 (1999).

3. C. Amelung, these proceedings.

4. S. Jindariani, these proceedings.

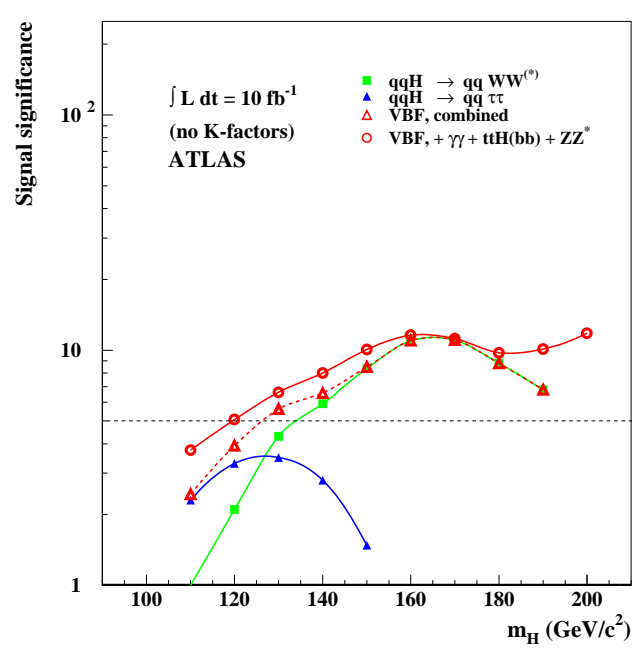

Figure 6. Significance of Higgs signals from different decay and production mechanisms as function of the Higgs mass $m_{H}$ in the low- $m_{H}$ region [17].

5. A. Moraes, C. Buttar, I. Dawson, ATLAS Note ATL-PHYS-PUB-2005-007.

6. T. Petersen, these proceedings.

7. T. Tait, these proceedings.

8. A. Tricoli, A. Cooper-Sarkar, C. Gwenlan, ATLAS Note ATL-PHYS-CONF-2005-008.

9. I. Borjanovic et al., ATLAS Scientific Note SN-ATLAS-2004-040 (2004).

10. A. Santocchia, these proceedings.

11. S. Bentvelsen, M. Cobal, ATLAS Note ATLPHYS-PUB-2005-024.

12. D.R. Tovey, EPJdirect C4, CN4, 124 (2002) and ATLAS Scientific Note SN-ATLAS-2002020 (2002).

13. M. Biglietti et al., ATL-PHYS-2004-011 (2004).

14. S. Rahatlou, these proceedings.

15. F. Ledroit, J. Morel, B. Trocmé, ATLAS Note ATL-PHYS-PUB-2006-024.

16. L. R. Flores Castillo, these proceedings.

17. S. Asai et al., ATLAS Scientific Note SNATLAS-2003-024 (2003). 